\title{
EchoGéo
}

21 | 2012

Pays émergents

\section{'Peace is a cup of coffee' : espaces publics dans Le Cap post-apartheid}

\section{Myriam Houssay-Holzschuch}

\section{OpenEdition}

1 Journals

Édition électronique

URL : https://journals.openedition.org/echogeo/13054

DOI : $10.4000 /$ echogeo.13054

ISSN : 1963-1197

\section{Éditeur}

Pôle de recherche pour l'organisation et la diffusion de l'information géographique (CNRS UMR 8586)

Référence électronique

Myriam Houssay-Holzschuch, «'Peace is a cup of coffee' : espaces publics dans Le Cap postapartheid », EchoGéo [En ligne], 21 | 2012, mis en ligne le 10 octobre 2012, consulté le 10 août 2021. URL : http://journals.openedition.org/echogeo/13054; DOI : https://doi.org/10.4000/echogeo.13054

Ce document a été généré automatiquement le 10 août 2021.

EchoGéo est mis à disposition selon les termes de la licence Creative Commons Attribution - Pas d'Utilisation Commerciale - Pas de Modification 4.0 International (CC BY-NC-ND) 


\title{
'Peace is a cup of coffee' : espaces publics dans Le Cap post-apartheid
}

\author{
Myriam Houssay-Holzschuch
}

\section{NOTE DE L'AUTEUR}

Cette contribution a bénéficié des travaux de l'ACI nJC2069 où les travaux sur Cape Town avaient été menés avec Annika Teppo (certains entretiens ayant été effectués par Jeanne Vivet) et de l'ANR PERISUDS nº Suds-07-046.

1 La démocratisation a changé les conditions de possibilité de l'espace public en Afrique du Sud et notamment de l'espace public de la co-présence (Houssay-Holzschuch, 2007, 2010; Sabatier 2010). Les années 1990 ont notamment été caractérisées par une croissance de la criminalité, conduisant à un déclin des espaces publics traditionnels (rues, parcs, places) (illustration 1) et à la multiplication des phénomènes de sécurisation résidentielle par la clôture (illustration 2) ou les communautés fermées (illustration 3) (Lemanski et al., 2008 ; Morange et al., 2006). 
Illustration 1 - SDF, Grand Parade, devant l'ancien hôtel de ville

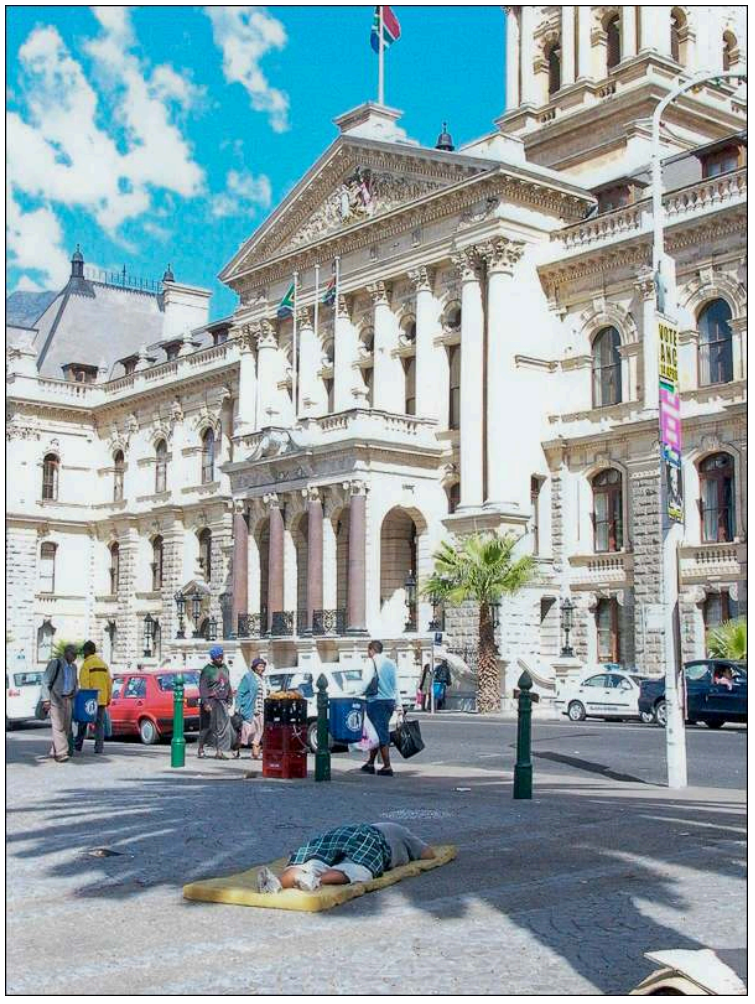

Auteur: Myriam Houssay-Holzschuch, Pâques 2001.

Illustration 2 - Epicerie de quartier, Summergreens, juillet 2005

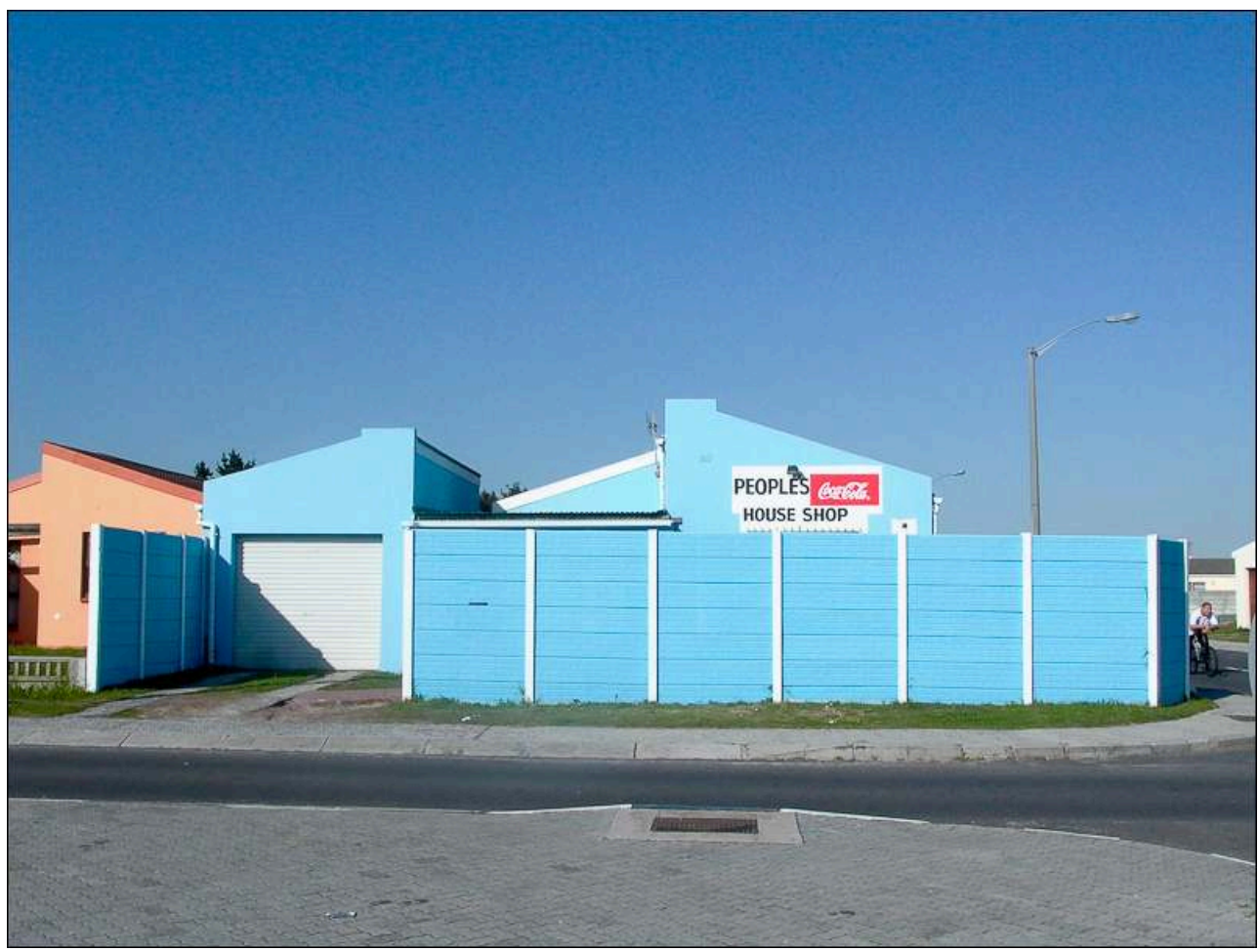

Auteur : Myriam Houssay-Holzschuch, juillet 2005. 
Illustration 3 - L'entrée de la communauté fermée de Steenberg, organisée autour de son golf et dans un vignoble renommé

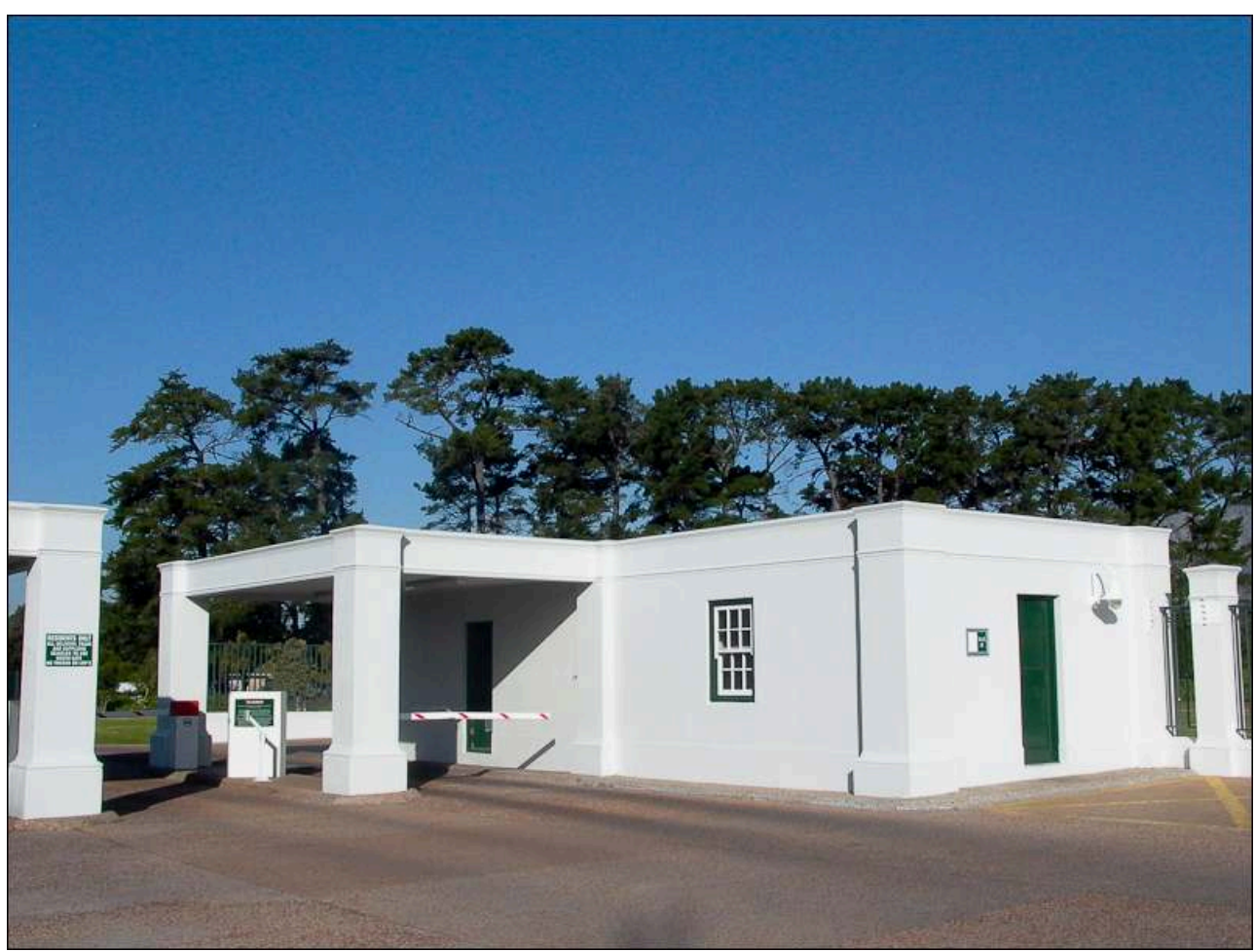

Auteur: Myriam Houssay-Holzschuch, juillet 2005.

2 Néanmoins tout espace peut devenir espace public, par son usage comme espace public (Mitchell, 2003). Le lieu le plus emblématique des nouveaux espaces communs en construction au Cap est un mall, le V\&A Waterfront (Houssay-Holzschuch \& Teppo, 2009; Houssay-Holzschuch \& Vivet, 2009). S'il incarne des processus de commercialisation, muséification, disneyilandification, communautarisation, privatisation, sécurisation..., il a surtout un public dont la composition raciale ${ }^{1}$ reflète celle de la ville et socialement très divers (illustration 5). 
Illustration 4 - Le V\&A Waterfront

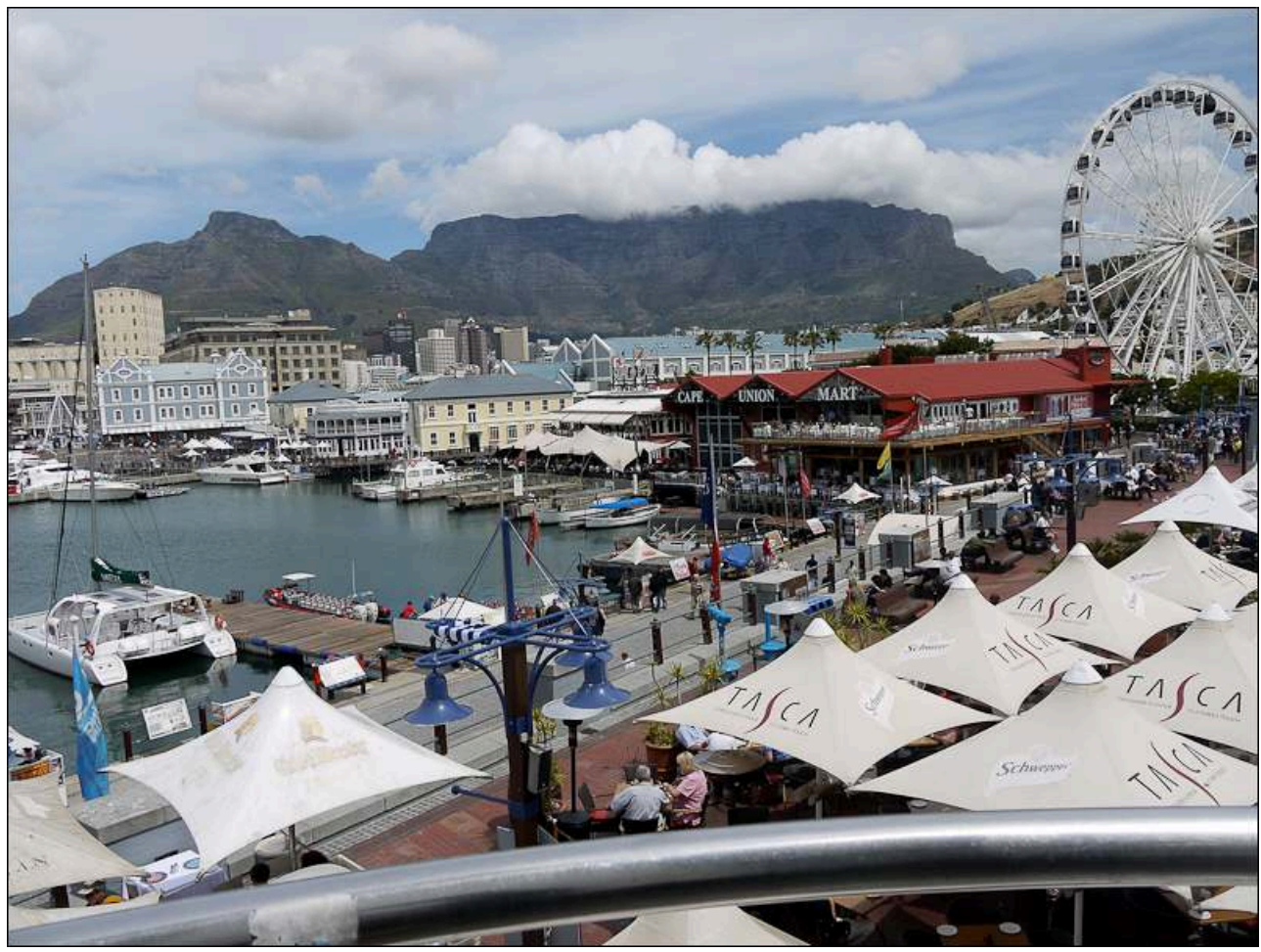

Auteur : Myriam Houssay-Holzschuch, octobre 2011

Illustration 5 - Maids et cleaning ladies Coloureds et africaines viennent au V\&A Waterfront le weekend, pour flâner, se reposer et profiter du spectacle de la " rue »

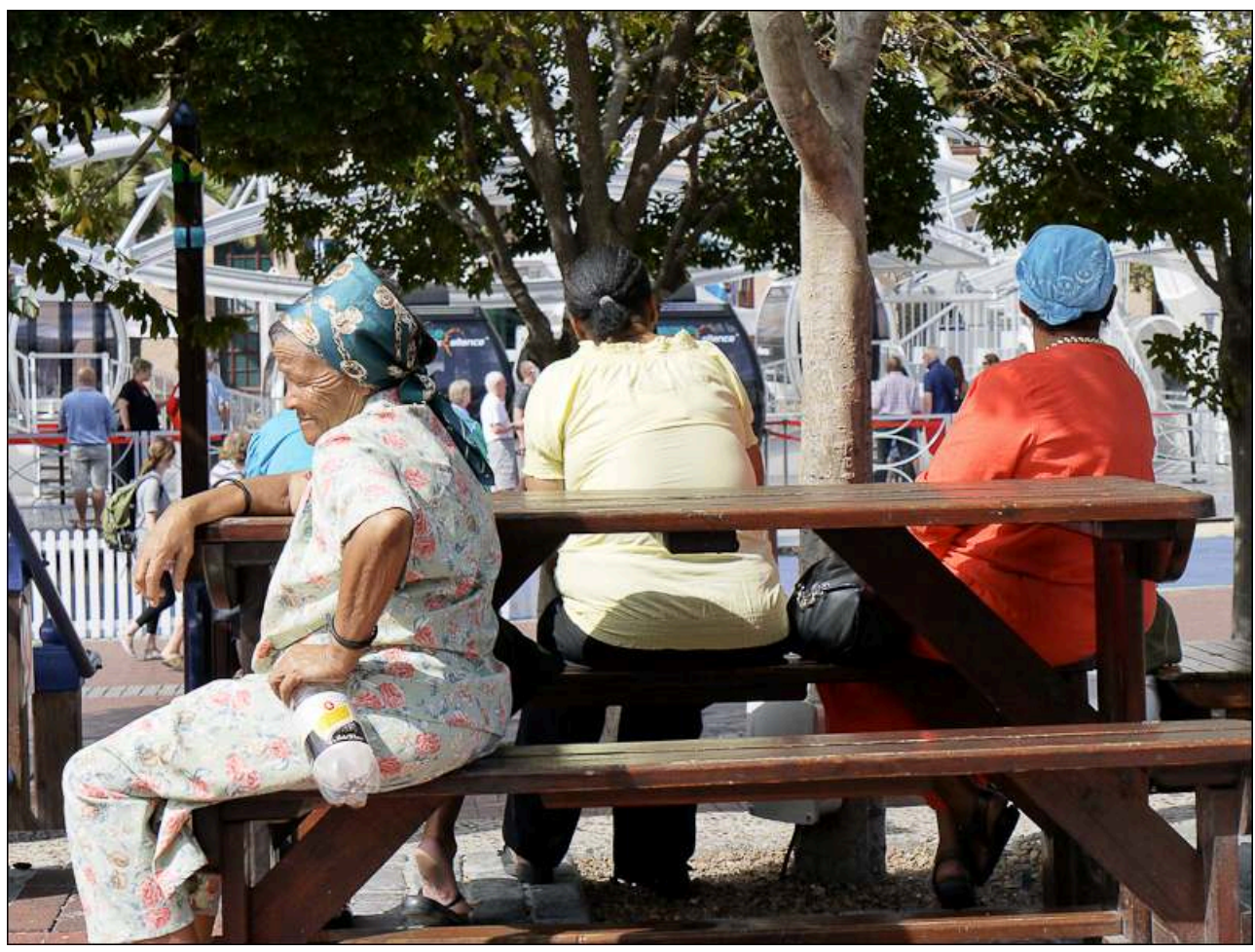

Auteur : Myriam Houssay-Holzschuch, mars 2011 
3 C'est un espace de co-présence, de cosmopolitisme dans une ville racialement et socialement très ségréguée :

«I like this place also because you can meet different people and you get to know them. (...) You can see different people, like in languages, in cultures, but they can meet each other. Yes it really reflects the new South Africa. » (agent de sécurité africain, 33 ans)

"The young people, they come to stand and look. They want to look pretty, and fashionable ... It is an extroverted place. (...) I like to look at the foreigners. » (homme, Coloured, 19 ans)

"I like the entertainments, like the music; and also you can see people who are in TV, you can see celebrity, famous politicians. » (agent de sécurité africain, 27 ans) " This place is a sort of entertainment. There are people from all over the world. » (chômeuse africaine, 53 ans)

" All the rain bow nation, it is mixed. (femme Coloured, project coordinator, 26 ans) "Yes, because you can see all kind of people and not one that dominate; it is easy to come here. » (étudiante Coloured, 22 ans) $»^{2}$

Illustration 6 - Couples mixtes, bande de copines coloured musulmanes, touristes blancs venus d'Europe, jeunesse des townships africains et enfants aux pieds nus se côtoient sous le regard attentif d'une sécurité très visible

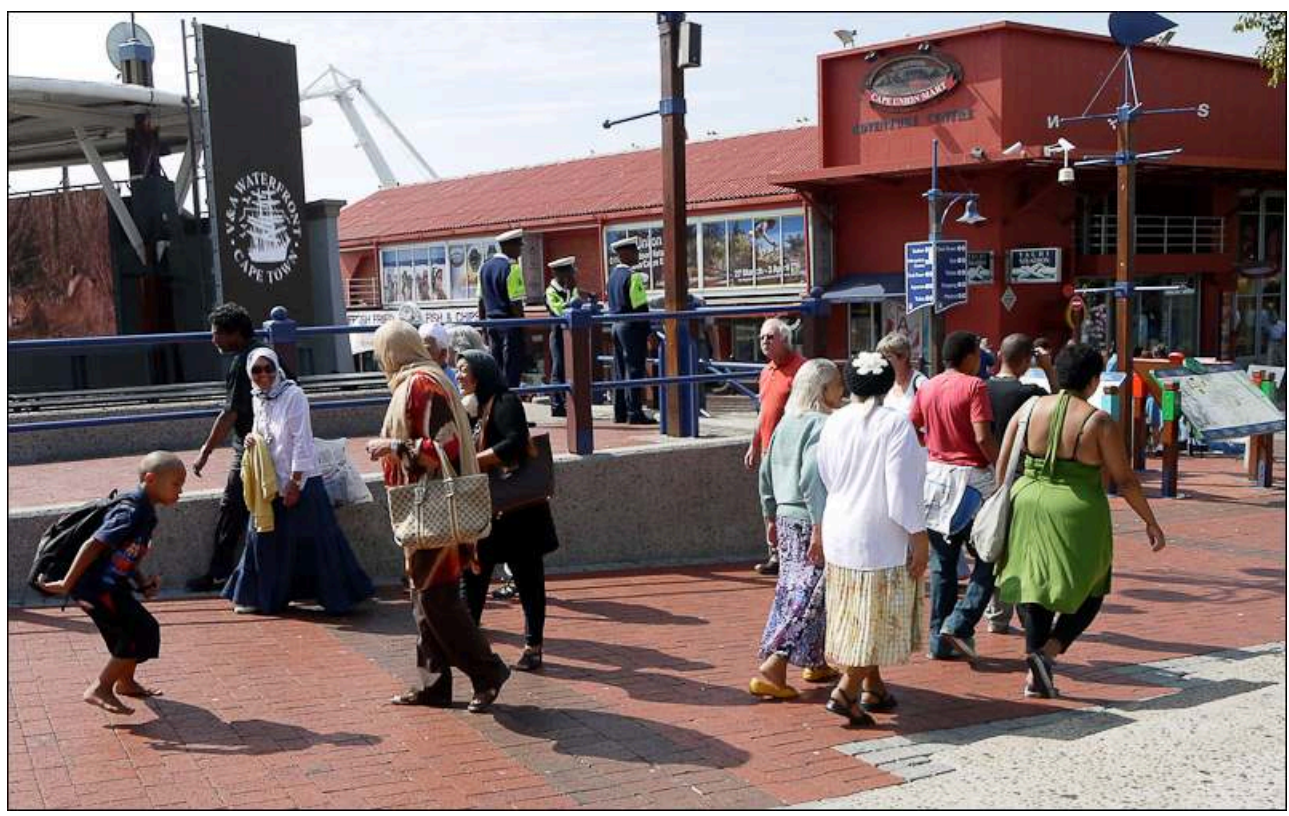

Auteur : Myriam Houssay-Holzschuch, mars 2011

4 Quant à la portée politique de tels lieux et de tels côtoiements éphémères (illustration 6) dans toute leur ambiguïté post-apartheid, elle ne peut mieux être exprimée que par ce vétéran de la branche armée de l'ANC, pour qui le lieu, son accessibilité nouvelle et sa civilité incarnent bien la paix et le projet inachevé d'une société plus juste :

«In different areas "peace" means different things. (...) So, peace in Paris and peace in Cape Town, they are different things but for me, peace means a cup of coffee where you can share a cup of coffee anywhere where you want to be. In the Waterfront, without any fear (...) - it's to afford that cup of coffee in these days you'll feel no discrimination and you will feel that I'm not occupying one space... this is my space too... and I've got a right to be there, as long as I can afford. And now the issue is to afford it. $»^{3}$ (LizoNtsabela, 28 juillet 2009, transcription Elsabe Jansen Van Vuuren) 


\section{BIBLIOGRAPHIE}

Houssay-Holzschuch M., 2007. Les espaces publics dans les pays intermédiaires. In Paris, Ministère de la Recherche et de l'Enseignement supérieur. http://hal.archives-ouvertes.fr/ hal-00591141_v1/

Houssay-Holzschuch M., 2010. Crossing boundaries : vivre ensemble dans l'Afrique du Sud post apartheid. HDR en géographie, Université Paris I Panthéon-Sorbonne, Paris. http://hal.archivesouvertes.fr/docs/00/55/10/98/PDF/HDRt1.pdf

Houssay-Holzschuch M., Teppo A., 2009. A mall for all ? Race and public space in post-apartheid Cape Town. Cultural geographies, 16, p. 351-379. http://hal.archives-ouvertes.fr/hal-00400561

Houssay-Holzschuch M., Vivet J., 2009. Blurring the line: privatisation and publicisation at the Victoria \& Alfred Waterfront, Cape Town. In C. Beni-Gbaffou, S. Fabiyi, E. Peyroux (Eds.), Sécurisation des quartiers et gouvernance locale : enjeux et défis pour les villes africaine (Afrique du Sud, Mozambique, Namibie, Nigéria), Johannesburg/Paris, IFAS/Karthala, p. 269-288.

Lemanski C., Landman K., Durington M., 2008. Divergent and Similar Experiences of "Gating" in South Africa: Johannesburg, Durban and Cape Town. Urban Forum, 19, p. 133-158.

Mitchell D., 2003. The right to the city; social justice and the fight for public space. New York, Guilford Press.

Morange M., Vivet J., 2006. Au Cap : peurs sur la ville ? In G. Capron, Quand la ville se ferme. Quartiers résidentiels sécurisés. Paris, Bréal, p. 34-57.

Sabatier B., 2006. La publicisation des espaces de consommation privés. Les complexes commerciaux récréatifs en France et au Mexique. Thèse de géographie, Université de Toulouse II Le Mirail. http:// tel.archives-ouvertes.fr/tel-00080769

\section{NOTES}

1. La classification raciale en vigueur sous l'apartheid comprenait les "Blancs ", Sud-Africains d'origine européenne ; les "Indiens ", Sud-Africains d'origine indienne ; les "Africains ", SudAfricains d'origine africaine ; les Coloureds, groupe hétérogène incluant les personnes ne rentrant pas dans les catégories précédentes. Cette classification joue toujours un rôle important dans la construction identitaire et sera donc employée selon lesconventions sud-africaines.

2. "J'aime ce lieu, parce qu'on peut y rencontrer des gens différents et faire leur connaissance. (...) On peut voir des gens différents, par leur langue, leurs cultures, mais ils peuvent se rencontrer. Oui, cela reflète vraiment la nouvelle Afrique du Sud.»

« Les jeunes, ils viennent traîner et voir. Ils veulent avoir l'air jolis et à la mode... C'est un endroit extraverti. (...) J'aime bien regarder les étrangers. »

"J'aime les attractions, comme la musique et aussi on peut voir des gens qui sont à la télé, on peut voir des célébrités, des hommes politiques célèbres. "

«Ce lieu est un genre d'attraction. Il y a des gens du monde entier.»

«Toute la nation arc-en-ciel, elle est mélangée. »

"Oui, parce qu'on peut voir toutes sortes de gens et pas un qui domine ; c'est facile de venir ici. »

3. «Dans des lieux différents, la 'paix' a des significations différentes. (...) Donc, la paix à Paris et la paix au Cap sont des choses différentes mais pour moi, la paix, c'est une tasse de café, quand tu peux partager une tasse de café à l'endroit que tu veux. Au Waterfront, sans aucune peur (...) - et 
pouvoir s'offrir cette tasse de café, aujourd'hui tu ne ressentiras aucune discrimination et tu ne trouveras pas que j'occupe un lieu... c'est aussi un lieu à moi... et j'ai le droit d'être là, tant que je peux me l'offrir. Et maintenant, le problème est de pouvoir se l'offrir.»

\section{AUTEUR}

\section{MYRIAM HOUSSAY-HOLZSCHUCH}

Myriam Houssay-Holzchuch est Professeur de géographie à l'Université Grenoble 1, PACTE UMR 5194 (CNRS, IEPG, UJF, UPMF) et membre de l' Institut Universitaire de France.

Myriam.Houssay@normalesup.org 